\title{
The dyonic picture of topological objects in the deconfined phase
}

\author{
V. G. Bornyakov, ${ }^{1,2}$ E.-M. Ilgenfritz, ${ }^{3,4}$ B. V. Martemyanov, ${ }^{2}$ and M. Müller-Preussker ${ }^{3}$ \\ ${ }^{1}$ Institute for High Energy Physics, Protvino, 142281, Russia \\ ${ }^{2}$ Institute for Theoretical and Experimental Physics, \\ B. Cheremushkinskaya 25, Moscow 117259, Russia \\ ${ }^{3}$ Institut für Physik, Humboldt-Universität zu Berlin, Newtonstr. 15, D-12489 Berlin, Germany \\ ${ }^{4}$ Institut für Physik, Karl-Franzens-Universität Graz, Universitätsplatz 5, A-8010 Graz, Austria
}

(Dated: March 3, 2022)

\begin{abstract}
In the deconfinement phase of quenched $S U(2)$ Yang-Mills theory the spectrum and localization properties of the eigenmodes of the overlap Dirac operator with antiperiodic boundary conditions are strongly dependent on the sign of the average Polyakov loop, $\langle L\rangle$. For $\langle L\rangle>0$ a gap appears with only few, highly localized topological zero and near-zero modes separated from the rest of the spectrum. Instead of a gap, for $\langle L\rangle<0$ a high spectral density of relatively delocalized near-zero modes is observed. In an ensemble of positive $\langle L\rangle$, the same difference of the spectrum appears under a change of fermionic boundary conditions. We argue that this effect and other properties of near-zero modes can be explained through the asymmetric properties and the different abundance of dyons and antidyons - topological objects also known to appear, however in a symmetric form, in the confinement phase at $T<T_{c}$ as constituents of calorons with maximally nontrivial holonomy.
\end{abstract}

PACS numbers: 11.15.Ha, 11.10.Wx

\section{INTRODUCTION}

The study of topological objects at nonzero temperature on the basis of smeared $S U(2)$ lattice fields [1] has suggested the following picture of the topological content of $S U(2)$ lattice gauge theory. At low temperatures topological objects are represented by nondissociated calorons with maximally nontrivial holonomy [2, 3, 4]. (see also Ref. [5]). With increasing temperature their composite nature becomes recognizable. They start to dissociate into dyons of topological charge $\pm 1 / 2$. Approaching the critical temperature $T_{c}$ (of the deconfining phase transition) from below, approximately 50 percent of the calorons become dissociated, retaining their symmetric properties. Above the critical temperature, a nonzero expectation value of the averaged Polyakov loop $\langle L\rangle \equiv\langle\overline{P(\vec{x})}\rangle$, where $\overline{P(\vec{x})}$ means the 3 -space average of the local values $P(\vec{x})$, is realized, apart from tunnelings changing the sign of $\bar{P}$ which are suppressed in large volumes. In the result there appears an asymmetry of dyons with peaked values of the local Polyakov loop $P(\vec{x})$ differing in sign: light dyons with the local Polyakov loop of same sign as $\langle L\rangle$ become the most abundant topological objects, while heavy dyons (and even more nondissociated calorons) are suppressed.

For the confinement phase, Diakonov and Petrov [6] have developed a confining dyon gas picture. Although it is a model either for purely selfdual (or antiselfdual) constituents, it explains all essential features of that phase. For the numerical success it is important that both selfdual and antiselfdual gases cooperate without interaction. This attractive model is, however, difficult to substantiate in lattice simulations because at low temperature the distances between caloron constituents are hard to be resolved.

Whether dissociated [6] or undissociated bound in calorons [7], dyons and antidyons in the confinement phase with $\langle L\rangle=0$ are completely symmetric concerning the abundance between all four sorts of constituents: selfdual dyons $M, L$ (with positive topological charge, i.e. equal-sign electric and magnetic charge) forming calorons and antiselfdual antidyons $\bar{M}, \bar{L}$ (with negative topological charge, i.e. opposite-sign electric and magnetic charge) forming anticalorons. This nomenclature was coined by Diakonov and Petrov [8] in a paper, where they considered the role of these Bogomol'nyi-PrasadSommerfield [9] dyons not in nonsupersymmetric YangMills theory but in $\mathcal{N}=1$ supersymmeric $S U(2)$ YangMills theory.

What do we actually know about single caloron solutions ? The semiclassical amplitude for $S U(2)$ calorons has been calculated in Ref. [10]. There it has been shown that they are stable above $T_{c} \approx \Lambda_{\mathrm{QCD}}$ for holonomy $|\langle L\rangle|>0.787597$. This actually leaves the room open for a dyon gas model as sketched above to describe the confined phase, but wasn't meant as an argument that the deconfined phase would be correctly described as a gas of "atomic" calorons and anticalorons.

A naive picture, assuming that undissociated calorons dominate the deconfined phase, would associate the onset of confinement with the dissociation of calorons. The high-temperature phase would be a gas of calorons in their undissociated form, with a radius decreasing with rising $T$ and adapted in shape to the respective $\langle L\rangle$. This point of view was taken in Ref. [7] as far as the deconfined phase was considered and found to disagree with the lattice observations. It ignores the possibility that the emerging nonzero value of the average Polyakov loop generates an enormous asymmetry between (anti) dyons with a local Polyakov loop $P(\vec{x})$ (in the center of their action or topological charge lumps) having different sign. If they were not necessarily bound 
in a caloron, "light dyons" with a peak value of the Polyakov loop $P(\vec{x})$ equal in sign to $\langle L\rangle$ could become the most abundant objects carrying only a small amount of topological charge, while "heavy dyons" with a local Polyakov loop $P(\vec{x})$ opposite in sign to $\langle L\rangle$ could be heavily suppressed while carrying a relatively large topological charge close to $|Q|=1$. Indeed, the higher action $S_{\text {heavy }}=\frac{16 \pi^{2}}{g^{2}}\left(\frac{1}{2}-\omega\right)$ (if $\langle L\rangle=\cos (2 \pi \omega)>0$ ) of the heavy dyons would be a natural explanation of their statistical suppression compared to light dyons with their lower action $S_{\text {light }}=\frac{16 \pi^{2}}{g^{2}} \omega$ (see section $\amalg$ for definition of $\omega)$.

The analysis of topological objects by means of overlap fermions has confirmed the observations made for smeared lattice fields at the temperature of the thermal phase transition [11]. Since that time, an extended and model-independent investigation of overlap fermion spectra for $S U(2)$ below and above $T_{c}$ (up to $T=2 T_{c}$ ) was performed [12]. The fermionic eigenmodes (and their spectral density) show some striking peculiarities above $T_{c}$ that our dyonic picture of the topological content of $S U(2)$ lattice gauge theory seems to be able to explain. In the time since our paper [1] was written, our own analysis of topological objects by means of overlap fermions has progressed and concentrated on the hightemperature phase, not only on properties of individual overlap modes, but also on the possibility to extract 13] topological properties of the gauge field. In this paper we present the analysis of topological clusters above $T_{c}$ which again requires to use the UV filtered definition of the topological density. In this respect, the present work is a direct continuation of the previous one on calorons and dyons at the thermal phase transition [11]. The knowledge of the spectral density and localization properties of individual modes, now detailed for both signs of $\langle L\rangle$ for fixed, antiperiodic temporal boundary conditions [12] or - vice versa - depending on the temporal boundary conditions for fixed $\langle L\rangle>0$ as considered in this paper ${ }^{1}$, corroborates the interpretation of the present cluster results that has emerged in the meantime.

In our previous work 11] concentrating on topological clusters we considered $S U(2)$ gluodynamics on $20^{3} \times 6$ lattice using the tree-level improved Symanzik action at $\beta_{\text {imp }}=3.25$ corresponding to the thermal phase transition. Here we use a lattice of size $20^{3} \times 4$ at the same $\beta_{\text {imp }}$, i.e. we are studying gluodynamics at a temperature $T=1.5 T_{c}$. Our analysis is based on 67 equilibrium configurations. For each configuration we have obtained the 20 lowest eigenmodes of the Dirac operator.

The paper is organized as follows: in Sect. II we analyze the properties of overlap fermion spectra above $T_{c}$

\footnotetext{
${ }^{1}$ It is clear that for the Dirac operator spectrum changing sign of the averaged Polyakov loop is equivalent to changing boundary conditions from antiperiodic boundary conditions to periodic boundary conditions.
}

as found in this paper and in Ref. [12] on the basis of the dyonic picture of the topological content of $S U(2)$ lattice gauge theory. In Sect. III we discuss the reason for the enormous difference in localization [12] of the eigenmodes in the gap region between the two types of boundary conditions or between the two signs of the average Polyakov loop, respectively. In Sect. IV we investigate topological clusters and their respective degree of (anti-)selfduality following the idea of Ref. [14 by constructing the topological charge density and the field strength tensor 13 in an UV filtered way from the lowest fermion eigenmodes. In Sect. $\mathrm{V}$-additional dyonic signatures of topological objects are presented, as those connected to the profiles of fermion modes, those connected to the monopole content of topological clusters and those related to the profiles of the Polyakov loop throughout the clusters. Finally, we conclude in Sect. VI.

\section{OVERLAP FERMION SPECTRA}

The classical caloron solution with nontrivial holonomy consists of two dyons with oppositely peaked values of the local Polyakov loop $P(\vec{x})$. This local field assumes a value equal to 1 or -1 [3] inside the constituents. The coincidence of the eigenvalues of the holonomy matrix is the definition of a monopole. For the caloron solution the overlap Dirac operator with periodic boundary conditions has a zero mode localized on the dyon with positive central Polyakov loop while the operator with antiperiodic boundary conditions has a zero mode localized on the dyon with negative central Polyakov loop [15, 16]. Whether the zero mode jumps from one dyon to another when the boundary conditions are changed [17] depends only on the degree of separation between the constituents (dissociation). Even within a nonseparated lump of action the zero mode is able to oscillate inside the lump under a change of boundary conditions.

A pair of dyon and antidyon with a same sign of the central Polyakov loop values $P\left(\vec{x}_{1}\right)$ and $P\left(\vec{x}_{2}\right)$ cannot constitute a classical solution. For field configuration having only topological objects of this type the Dirac operator has no exact zero mode because the total topological charge is zero. If both $P\left(\vec{x}_{i}\right)>0$ or both $<0$, the Dirac operator with periodic (antiperiodic) boundary conditions has two near-zero modes which tend to become zero modes (of opposite chirality) only in the limit of infinite dyon-antidyon separation. This can be seen in Fig. 1. where the spectrum for an artificially constructed dyon-antidyon pair is shown. A similar pair has been obtained from generic lattice configurations by overimproved cooling in Ref. [18] and shown in Fig. 11 therein.

Now let us compare the fermion spectrum for such an artificial dyon-antidyon pair with those of equilibrium Monte-Carlo configuration in the deconfined phase of pure $S U(2)$ lattice gauge theory. This is shown in Fig. 2 for periodic boundary conditions and antiperiodic boundary conditions. The Monte Carlo configuration is a typical configuration from a sample with Polyakov loop 
$\langle L\rangle>0$. It can be seen from Fig. 2 that the spectra for periodic boundary conditions and antiperiodic boundary conditions are very different. For periodic boundary conditions the spectrum has no gap while for antiperiodic boundary conditions there is a wide gap. Guided by the similarity between Fig. 1; and Fig. 2 we can propose the following explanation of this difference. In the case of configurations with $\langle L\rangle>0$ light dyon-antidyon pairs with a Polyakov line peaking at $P(\vec{x})=+1$ appear in a large number and give rise to numerous near-zero modes in the spectrum of the Dirac operator with periodic boundary conditions. This eliminates the spectral gap completely, while in the case of the Dirac operator with antiperiodic boundary conditions only heavy dyonantidyon pairs can produce near-zero modes, and such pairs are rare. This is reflected by the known fact of a gap opening for the case of antiperiodic boundary conditions. In fact, in equilibrium configurations there is a small number of (exceptional) near-zero modes seen. This has been first discovered in [19] and recently confirmed in [12]. With increasing temperature, they become more and more separated from the rest of the spectrum (bulk) by the emerging gap and are decreasing in multiplicity [12].

We identify the number of near-zero modes $n_{\mathrm{nzm}}$ $\left(N_{\text {nzm }}\right)$ found for periodic (antiperiodic) boundary conditions (both with $\langle L\rangle>0$ ) with the number of light (heavy) dyon-antidyon pairs. The ratio of $n_{\mathrm{nzm}} / N_{\mathrm{nzm}}$ can be estimated from Fig 3 where the spectra of 20 lowest eigenmodes are shown for both boundary conditions. Choosing the cut on near-zero modes as $|\operatorname{Im} \lambda|<$ $0.05 / a \approx 100 \mathrm{MeV}$ shown on the Fig 3 by the vertical dashed line we get $n_{\mathrm{nzm}} / N_{\mathrm{nzm}} \approx 15$. This number cannot be exact because in the case of periodic boundary conditions near-zero modes are not clearly separated by a gap from the rest of the spectrum (the bulk). Therefore some of the modes counted in $n_{\text {nzm }}$ might actually belong to the bulk. With this reservation in mind, one could conclude from the observed in Ref. [12] increasing with temperature spectral density of the periodic Dirac operator that the ratio $n_{\mathrm{nzm}} / N_{\mathrm{nzm}}$ is also increasing with temperature.

An alternative estimate for the number of light dyonantidyon pairs, $n_{\mathrm{nzm}}$, can be made using the following observation. In a dilute gas of light and heavy dyons ${ }^{2}$ each object independently contributes to the square of the topological charge $Q^{2}$ such that the sum over all configurations can be presented by

$$
\sum Q^{2}=q^{2} n+(1-|q|)^{2} N
$$

where $n=n_{\mathrm{zm}}+n_{\mathrm{nzm}}$ is the total number of zero and near-zero modes for the Dirac operator with periodic

\footnotetext{
${ }^{2}$ In the following "dyon" will be used without difference for carriers of positive and negative topological charge.
}

boundary conditions, i.e. the total number of light dyons summed over all configurations, and $N=N_{\mathrm{zm}}+N_{\mathrm{nzm}}$ is the total number of zero and near-zero modes for the Dirac operator with antiperiodic boundary conditions, i.e. the total number of heavy dyons summed over all configurations. Thereby, $|q|$ is a fractional topological charge of a light dyon and the complement $1-|q|$ that of a heavy dyon. We remind that we are discussing an ensemble with positive averaged Polyakov loop $\langle L\rangle>0$ which is actually the case for the configurations analyzed in the present paper. For our set of 67 configurations we find that $\sum Q^{2}=78, N_{\mathrm{zm}}=52, N_{\mathrm{nzm}}=30$, and $n_{\mathrm{zm}}=54$. We estimate the ratio between the topological charges of light and heavy dyons by the formula

$$
|q|=2 \omega
$$

known from the analytical caloron solution:

$$
|q|:(1-|q|)=\omega:(1 / 2-\omega),
$$

where $\omega$ is the parameter of holonomy $H=\cos (2 \pi \omega)$, and we identify the holonomy $H$ with the value of the average Polyakov loop $\langle L\rangle \approx 0.3$. We obtain $|q| \approx 0.4$ and $n_{\mathrm{nzm}} \approx 250$. Another estimate is based on the number $|q| \approx 0.3$ that correspond to the maximum of the heavy dyon mass distribution $1-|q| \approx 0.7$ (see Sect $\square$ ). Then $n_{\mathrm{nzm}} \approx 360$. These give a ratio $8.3<n_{\mathrm{nzm}} / N_{\mathrm{nzm}}<$ 12 in acceptable agreement with the value 15 obtained above comparing the number of low lying modes below $100 \mathrm{MeV}$ (see Fig [3), especially if one takes into account that we could have overestimated the number $n_{\mathrm{nzm}}$ of light nonzero modes in the case of periodic boundary conditions.

\section{LOCALIZATION OF EIGENMODES}

Next we turn to the issue of localization. This is a very natural question to ask in an investigation of the fermionic spectrum and eigenmodes. It turns out, that - adopting the view in terms of topological charge clusters - we can also contribute to the understanding of the strong dependence of the localization of zero and nearzero eigenmodes on the boundary conditions that was discovered in [12].

We find that for zero modes, the average inverse participation ratio (IPR) of antiperiodic zero modes is equal to 110 , while the IPR of periodic zero modes is equal to 4.75. For positive $\langle L\rangle$ - as in our case - the stronger localization of antiperiodic zero modes compared to periodic ones is easy to understand in the dyonic picture of the deconfined phase.

Each zero mode out of our $N_{\mathrm{zm}}=52$ antiperiodic zero modes is accompanied on average by $N_{\mathrm{nzm}} /\left(2 N_{\mathrm{zm}}\right) \approx 0.3$ pairs of antiperiodic near-zero modes, whereas the corresponding ratio for periodic boundary conditions according to the actual number $n_{\mathrm{zm}}=54$ of periodic zero modes requires to extract from (10) the number of periodic nearzero modes. Assuming $|q| \approx 0.4$ the ratio is obtained as 


\begin{tabular}{|c|c|c|c|c|c|c|}
\hline type & $P$ & $Q$ & $e$ & $m$ & action $\propto$ & size $\propto$ \\
\hline$M$ & +1 & $>0$ & \pm & \pm & $\omega$ & $(1 / 2-\omega)$ \\
$L$ & -1 & $>0$ & $\mp$ & $\mp$ & $(1 / 2-\omega)$ & $\omega$ \\
$\bar{M}$ & +1 & $<0$ & \pm & $\mp$ & $\omega$ & $(1 / 2-\omega)$ \\
$\bar{L}$ & -1 & $<0$ & $\mp$ & \pm & $(1 / 2-\omega)$ & $\omega$ \\
\hline
\end{tabular}

TABLE I: Local Polyakov loop $P$, sign of topological charge $Q$, electric $(e)$ and magnetic $(m)$ charge of dyons and antidyons. Action and size are given for positive external Polyakov loop $\langle\overline{P(\vec{x})}\rangle=\cos (2 \pi \omega)>0$.

$n_{\mathrm{nzm}} /\left(2 n_{\mathrm{zm}}\right) \approx 2.3$. According to our dyonic picture we can say that an antiperiodic zero mode is spread out on average over $1.3=1+0.3$ identical (i.e. with the same sign of topological charge) heavy dyons, while a periodic zero mode is spread out on average over $3.3=1+2.3$ identical light dyons. Moreover, taking into account that the radii of light and heavy dyons are different and follow, correspondingly to a caloron formula, the proportionality rule $r: R=(1-|q|):|q| \approx 1.5$, we shall expect that an antiperiodic zero mode is $\left(\frac{3.3}{1.3}\right) \cdot(1.5)^{3} \approx 9$ times more localized than a periodic zero mode. Repeating this estimate assuming $|q| \approx 0.3$ the above localization ratio is replaced by 43 . The actual ratio of the average IPR between antiperiodic zero modes and periodic zero modes for our ensemble, $110 / 4.75$, is halfway between the two estimates.

In Ref. [12] a tendency has been found that the localization of antiperiodic zero modes together with $\langle L\rangle>0$ is increasing with increasing temperature, while the localization of antiperiodic zero modes in the presence of $\langle L\rangle<0$ or, equivalently, periodic zero modes in the presence of $\langle L\rangle>0$, is decreasing with increasing temperature. This qualitatively corresponds to the dyonic picture where the number ratio between heavy and light objects (dyons and antidyons) decreases and the size ratio between light and heavy objects increases with increasing temperature. This tendency is incorporated in the results obtained here by the temperature dependence of $\omega$ and $q$.

The properties of the above mentioned $M, L, \bar{M}$ and $\bar{L}$ dyons and antidyons are summarized with respect to the dependence on $\omega$ in Table $\Pi$.

\section{TOPOLOGICAL CLUSTERS}

Now let us turn to the properties of clusters with respect to the UV filtered definition of the topological charge density. We remind the reader that we are considering here an ensemble of lattice configurations with average Polyakov loop $\langle L\rangle>0$. The fermionic definition 20] has an UV filtered variant 21, 22]. Both have an a priori ambiguity with respect to the fermionic bound- ary conditions:

$$
q_{\lambda_{\text {cut }}}^{(b)}(x)=-\sum_{\left|\lambda_{b}\right| \leq \lambda_{\text {cut }}}\left(1-\frac{\lambda_{b}}{2}\right) \psi_{\lambda_{b}}^{(b) \dagger}(x) \gamma_{5} \psi_{\lambda_{b}}^{(b)}(x),
$$

with $b=p$ denoting periodic and $b=a$ denoting antiperiodic temporal boundary conditions. Although the total topological charge given by the number of zero modes is not affected by the boundary condition as long as some smoothness properties of the gauge field are fulfilled [11], we find that the filtered density function really depends on the boundary condition $b$. This has allowed us in Ref. [1] to investigate the dyonic vs. caloron structure for $T \lesssim T_{c}$ by measuring the amount of displacement of constituents with different sign of $P(\vec{x})$.

For the high-temperature phase, we adopt one more difference in our procedure, depending on the type of boundary conditions. In the case of periodic boundary conditions we include all the 20 lowest modes which we computed. As discussed above, in this case the nearzero modes cannot be strictly separated from the bulk of the spectrum. In the case of antiperiodic boundary conditions, however, we include only the zero and near-zero modes in the definition (4) because they can be separated without any ambiguity from the bulk.

The antiperiodic boundary condition highlights the heavy constituents with negative local Polyakov loop, whereas the periodic boundary condition emphasizes the complementary light constituents with positive local Polyakov loop. In the same way as in Ref. 11] we now define clusters of topological charge as connected sets of lattice sites where the absolute value of topological charge density exceeds some cut, $|q(x)|>q_{\text {cut }}=\frac{1}{5} \max _{x}(|q(x)|)$. The density $q(x)=q_{\lambda_{\text {cut }}}^{(b)}(x)$ is the adopted version of UV filtered topological density defined by the respective selection of the modes ${ }^{3}$.

Furthermore, we have constructed from the fermionic modes the field strength tensor [23]

$$
c^{T} F_{\mu \nu}^{a}(x)=\sum_{\left|\lambda_{b}\right| \leq \lambda_{\text {cut }}} \lambda_{b} \psi_{\lambda_{b}}^{(b) \dagger}(x) \sigma_{\mu \nu} \tau^{a} \psi_{\lambda_{b}}^{(b)}(x),
$$

where $c^{T}=0.0883$ [24] has been calculated for the full spectrum. For $F_{\mu \nu}^{a}$ on the left hand side of Eq. (5) we have omitted the labels $b$ and $\lambda_{\text {cut }}$ in order to avoid clumsy formulae in what follows.

The topological charge densities defined by (4) on one hand and by the field strength tensor on the other, through the scalar product $\vec{E}^{a} \cdot \vec{B}^{a}$ of electric and magnetic field strength,

$$
q^{F}(x)=\frac{1}{32 \pi^{2}} F_{\mu \nu}^{a}(x) \tilde{F}_{\mu \nu}^{a}(x)
$$

\footnotetext{
3 The tail of the topological charge distribution with $|q(x)|<$ $\frac{1}{5} \max _{x}(|q(x)|)$ is part of the volume where we expect the field strength tensor to deviate strongly from being selfdual or antiselfdual.
} 
differ for truncated sums over modes like in our case by many orders of magnitude. However, if suitably rescaled, the density (6) closely follows the density (4). Fig. 4 shows this for an on-axis sequence of lattice sites in one particular configuration studied with periodic boundary conditions and taking all 20 modes into account. A rescaling factor $r$ has been found by minimizing the quantity

$$
\Delta^{2}=\overline{\left(q_{\lambda_{\text {cut }}}^{(b)}(x)-r \cdot q^{F}(x)\right)^{2}},
$$

where the bar means averaging over all lattice sites. We found that the parameter $r$ is of the order $O\left(10^{8}\right)$. For the particular configuration presented in Fig. 4 we have found the deviation $\Delta \approx 0.04 \max _{x}\left|q_{\lambda_{\text {cut }}}^{(b)}(x)\right|$, i.e. it amounts to only a few percent of the maximal density.

In order to focus on the dyonic nature of the topological objects detected by the respective number of modes, we tested the degree of (anti)selfduality [14] of the topological clusters as mapped out by the topological density (41). Site by site we considered the quantity

$$
R=\frac{4}{\pi} \arctan \frac{F_{\mu \nu}^{a} \cdot F_{\mu \nu}^{a}-F_{\mu \nu}^{a} \cdot \tilde{F}_{\mu \nu}^{a}}{F_{\mu \nu}^{a} \cdot F_{\mu \nu}^{a}+F_{\mu \nu}^{a} \cdot \tilde{F}_{\mu \nu}^{a}}-1 .
$$

Analogously to studies of the local chirality of fermionic modes, this quantity equals to $-1(+1)$ for a strictly selfdual (antiselfdual) field strength tensor. The distribution of this quantity for all lattice sites on one hand and restricted to the interior of the topological charge clusters on the other is shown in Fig. 5 The case of periodic boundary conditions, where the near-zero modes cannot be clearly separated from the background, is presented in the left panels of Fig. 55 the interior of the topological charge clusters (shown left below) is indeed preferentially selfdual or antiselfdual. In contrast, taking all lattice sites into account (shown left above), the distribution with respect to the degree of (anti)selfduality can hardly be distinguished from that obtained for a random assignment. This random reference case is represented by the fat line (red in the colored version) in the left panels of Fig. [5. The random construction consists in replacing $F_{\mu \nu}^{a} \cdot \tilde{F}_{\mu \nu}^{a}$ by a random number $q$ sampled from the interval $[-s,+s]$ with $s=F_{\mu \nu}^{a} \cdot F_{\mu \nu}^{a}$.

In the case of antiperiodic boundary conditions we are able to unambiguously restrict ourselves to the zero and near-zero modes in the construction of (4) and (5). From the right panels of Fig. 5 it becomes obvious now why we associate these modes to the heavy dyons (again irrespective of the sign of the topological charge density). Their contributions to (4) and (5) form what we can call "selfdual and antiselfdual heavy dyons". If the construction is limited to these modes the obtained field strength is constrained to be either selfdual or antiselfdual, irrespective whether we consider all lattice sites (shown right above) or only the interior of the clusters of sufficiently large topological charge density compared to the maximal density (shown right below). This is expressed by the fact that the right panels of Fig. 5 both collapse to a sum of $\delta$-functions at $R=-1$ and $R=+1$. The height of the peaks reflects the ratio of positive and negative topological charges summed over the ensemble. For this case all $N_{\mathrm{zm}}+N_{\mathrm{nzm}}=52+30=82$ heavy zero and near-zero modes are in one-to-one correspondence to the observed clusters of topological charge.

\section{PROFILES OF NEAR-ZERO AND ZERO MODES}

Last but not least we consider more in detail the shapes of the 82 heavy zero and nonzero fermionic modes in comparison to analytical results available for dyons [15]. Analytically, from the caloron solution, the profile of a zero mode localized on one of the constituent dyons is known to depend on two parameters of the caloron: the holonomy parameter $\omega$ that defines the "mass" fraction of the constituent (also the fractional topological charge) as $m_{h}=1-2 \omega$ and the distance $d$ from the center of the accompanying complementary constituent with a "mass" fraction $m_{l}=2 \omega$. Both parameters influence the nonstaticity of zero mode. The parameters $\omega$ and $d$ were found for every lattice zero and near-zero mode using the following procedure.

We evaluated the summed scalar density $\rho(t, \vec{x})$ of all zero and nonzero modes. There was a number of clusters of this density exactly equal to the number of zero and near-zero modes. First in each cluster the absolute maximum was found at $\vec{x}_{\text {max }}$ :

$$
\rho_{\max }=\max _{t} \max _{\vec{x}} \rho(t, \vec{x}) .
$$

Then in the same spatial point $\vec{x}_{\max }$ the minimum with respect to $t$ was determined:

$$
\rho_{\min }=\min _{t} \rho\left(t, \vec{x}_{\max }\right) .
$$

From the analytical expression for the zero mode of a one-caloron solution [15] the maximum (9) and the minimum values (10) of its scalar density can be derived and expressed as functions of parameters $\omega$ and $d$. Using these functions we determined the parameters from the values of $\rho_{\max }$ and $\rho_{\min }$ observed for the individual maxima of the scalar density that are considered as being related to exact zero modes or to zero modes that are mixed to form the pairs on near-zero modes. In this way we have obtained the distribution of the 82 antiperiodic ("heavy") fermionic modes over their mass fraction $m_{h}$. The corresponding histogram is presented in Fig. 6. Note that for most of the distribution $m_{h}>0.5$, and that the maximum is located at about 0.7 which approximately reconfirms the value $m_{h}=0.6$ obtained in section [I under the assumption that the holonomy is given by $\langle L\rangle$. The typical profile of a fermionic mode, represented by its scalar density taken at $t=t_{\max }, x=x_{\max }$, and $y=y_{\max }$ as a function of $z$ is shown in Fig. 7 together with the best-fitting profile of the analytically given scalar density of a fermion zero mode. 
A more complete description of the dyonic structure of the topological objects found with the help of periodic and antiperiodic fermionic modes can be given in terms of gluonic observables. From the analytical solution we know that dyons are magnetic monopoles and that the Polyakov loop is peaked with a positive sign of $P(\vec{x})$ if the dyon supports a periodic fermion zero mode and with a negative sign of $P(\vec{x})$ if the dyon supports an antiperiodic fermion zero mode [2, 15]. The gluonic configurations of the present investigation have been subject to smearing as described in Ref. [11], by 10 steps of APE smearing [25]. We made sure that this procedure does not change the low lying spectrum of overlap fermions. After smearing the Abelian magnetic monopole content and the Polyakov loop profile of the topological clusters have been recorded. Clusters with static Abelian monopoles occupy about $3 \%$ of the lattice volume and contain about $50 \%$ of all timelike Abelian monopole links. They have peaked values of the Polyakov loop correlated in sign with the fermionic boundary condition, in total agreement with the required dyon properties. These clusters are recorded in Fig. 8 in form of a scatter plot with respect to the maximal value of the topological charge density and the extremal value of the local Polyakov loop $P(\vec{x})$ inside the cluster (both including the sign). One can see that all heavy dyon clusters, denoted by triangles, have negative $P(\vec{x})$ and relatively large values of the maximal topological charge density. The points representing light dyon clusters are concentrated with their $P(\vec{x})$ close to +1 with a maximal topological charge density (here in terms of the gluonic topological charge density) not exceeding $\left|q_{L}(x)\right|=0.01$.

\section{CONCLUSIONS}

We presented in this paper new evidence for the dyonic nature of the topological fluctuations, this time for the deconfinement phase of $S U(2)$ lattice gauge theory. We show that the dyonic picture suggests an explanation of the strong difference in the spectrum of the overlap
Dirac operator with periodic and antiperiodic boundary conditions, which is the only explanation given to this phenomena so far. Furthermore, we show that the abundances of near-zero modes, the localization properties of zero modes and near-zero modes, the (anti)selfduality properties of topological clusters, the profiles of fermionic modes, the monopole content and the Polyakov loop profiles of topological clusters are in reasonable agreement with the dyonic picture of the topological objects in the "vacuum" above $T_{c}$, where light dyons (and light antidyons) are most abundant and heavy dyons (and heavy antidyons) are suppressed. All of these topological clusters have properties known from asymmetric caloron solution with only slightly nontrivial holonomy. Maximally nontrivial holonomy is realized only in the confinement phase, with a full symmetry between all types of dyons and antidyons. We have considered in particular a temperature $T=1.5 T_{c}$ where the average Polyakov loop determines the deviation from both limits of trivial and maximally nontrivial holonomy. We expect that at higher temperatures the asymmetry between light and heavy (anti)dyons in the vacuum will further increase. Asymptotically, the topological objects in the vacuum will be exclusively light dyons and antidyons appearing in equal number.

\section{Acknowledgements}

This work was partly supported by the DFG grant 436 RUS 113/739/0-2 together with the RFBR-DFG grant 06-02-04010. Two of us (V.G. B. and B.V. M. ) gratefully appreciate the support of Humboldt-University Berlin where this work was carried out to a large extent. V.G. B. is supported by grants RFBR 08-02-00661 and RFBR -7-02-00237a. E.-M. I. was supported by DFG (FOR 465 / Mu932/2). He is grateful to the Karl-FranzensUniversität Graz for the guest position he holds while the paper is being completed. We thank Falk Bruckmann and Christof Gattringer for comments on a draft version of this paper.
[1] E.-M. Ilgenfritz, B. V. Martemyanov, M. MüllerPreussker, and A. I. Veselov, Phys. Rev. D73 (2006) 094509.

[2] T. C. Kraan and P. van Baal, Phys. Lett. B435 (1998) 389.

[3] T. C. Kraan and P. van Baal, Nucl. Phys. B533 (1998) 627.

[4] K. Lee and C. Lu, Phys. Rev. D58 (1998) 025011.

[5] R. C. Brower, D. Chen, J. W. Negele, K. Orginos, and C.-I. Tan, Nucl. Phys. Proc. Suppl. 73 (1999) 557.

[6] D. Diakonov and V. Petrov, Phys. Rev. D76 (2007) 056001.

[7] P. Gerhold, E.-M. Ilgenfritz, M. Müller-Preussker, Nucl. Phys. B760 (2007) 1.

[8] D. Diakonov and V. Petrov, Phys. Rev. D67 (2003)
105007.

[9] M. K. Prasad and C. M. Sommerfield, Phys. Rev. Lett. 35 (1975) 760; E. B. Bogomol'nyi, Yad. Fiz. 24 (1976) 861 [Sov. J. Nucl. Phys. 24 (1976) 449].

[10] D. Diakonov, N. Gromov, V. Petrov, and S. Slizovskiy, Phys. Rev. D70 (2004) 036003.

[11] V. G. Bornyakov, E.-M. Ilgenfritz, B. V. Martemyanov, S. M. Morozov, M. Müller-Preussker, and A. I. Veselov, Phys. Rev. D76 (2007) 054505 .

[12] V. G. Bornyakov, E. V. Luschevskaya, S. M. Morozov, M. I. Polikarpov, E.-M. Ilgenfritz, and M. MüllerPreussker, arXiv:0807.1980, Phys. Rev. D79 (2009) (in print).

[13] E.-M. Ilgenfritz, K. Koller, Y. Koma, G. Schierholz, T. Streuer, and V. Weinberg, Phys. Rev. D76 (2007) 
034506.

[14] C. Gattringer, Phys. Rev. Lett. 88 (2002) 221601.

[15] M. Garcia Perez, A. Gonzalez-Arroyo, C. Pena, and P. van Baal, Phys. Rev. D60 (1999) 031901(R).

[16] M. N. Chernodub, T. C. Kraan, and P. van Baal, Nucl. Phys. Proc. Suppl. 83 (2000) 556.

[17] C. Gattringer and S. Schaefer, Nucl. Phys. B654 (2003) 30.

[18] F. Bruckmann, E.-M. Ilgenfritz, B. V. Martemyanov, and P. van Baal, Phys. Rev. D70 (2004) 105013.

[19] R. G. Edwards, U. M. Heller, J. E. Kiskis and R. Narayanan, Phys. Rev. D61 (2000) 074504.

[20] F. Niedermayer, Nucl. Phys. Proc. Suppl. 73 (1999) 105.

[21] I. Horvath, S. J. Dong, T. Draper, F. X. Lee, K.-
F. Liu, J. B. Zhang, and H. B. Thacker, Nucl. Phys. Proc. Suppl. 119 (2003) 688.

[22] I. Horvath, S. J. Dong, T. Draper, F. X. Lee, K.-F. Liu, N. Mathur, H. B. Thacker, and J. B. Zhang, Phys. Rev. D68 (2003) 114505.

[23] K.-F. Liu, A. Alexandru, and I. Horvath, Phys. Lett. B659 (2008) 773.

[24] A. Alexandru, I. Horvath, and K.-F. Liu, Phys. Rev. D78 (2008) 085002.

[25] T. DeGrand, A. Hasenfratz, and T. G. Kovacs, Nucl. Phys. B520 (1998) 301. 


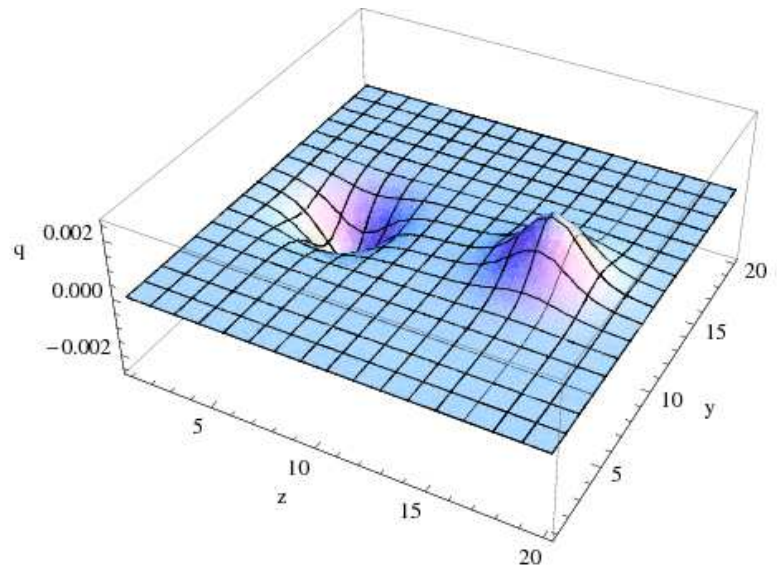

(a)

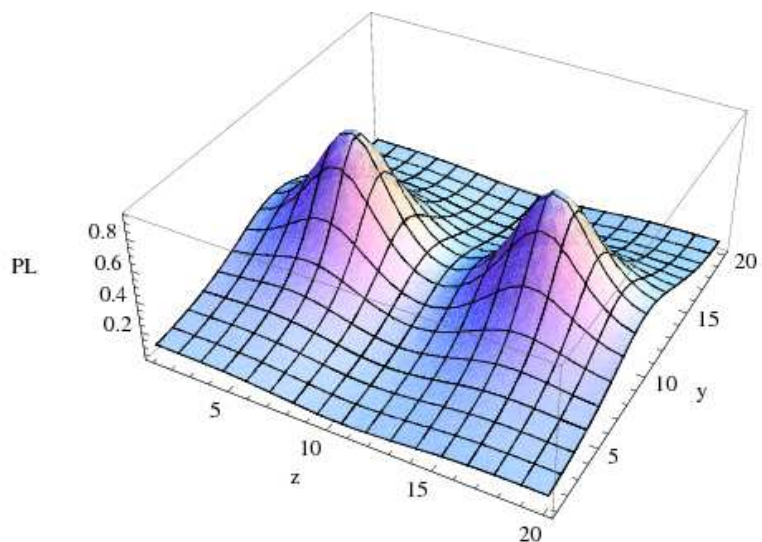

(b)

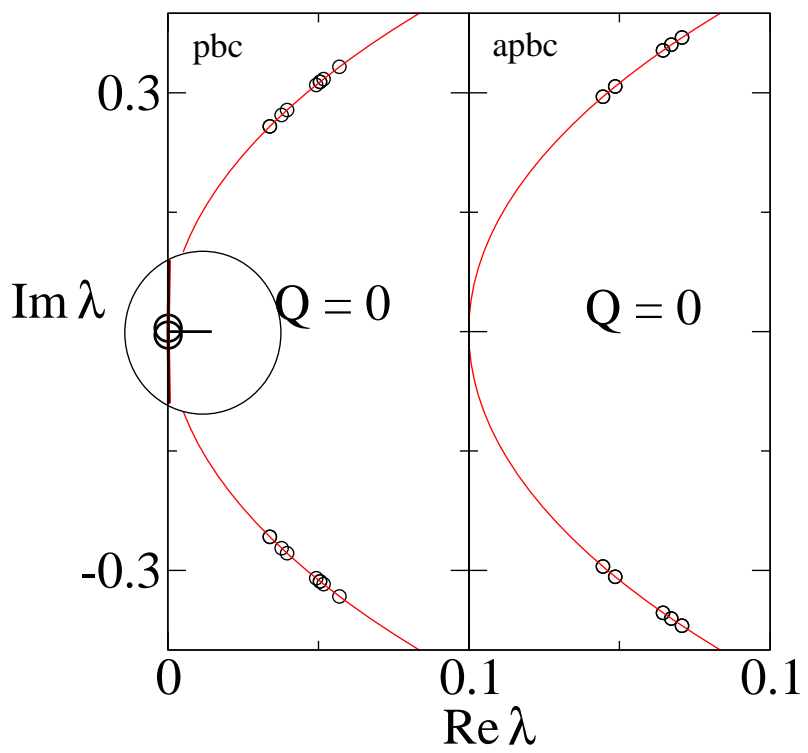

(c)

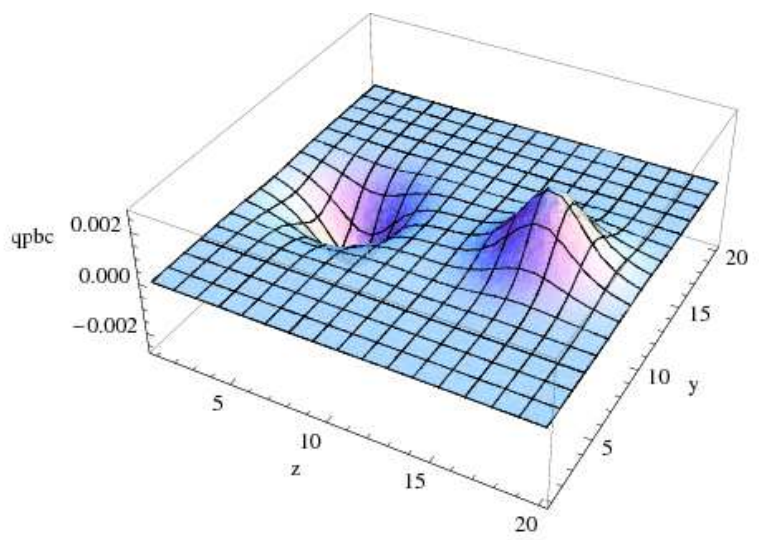

(d)

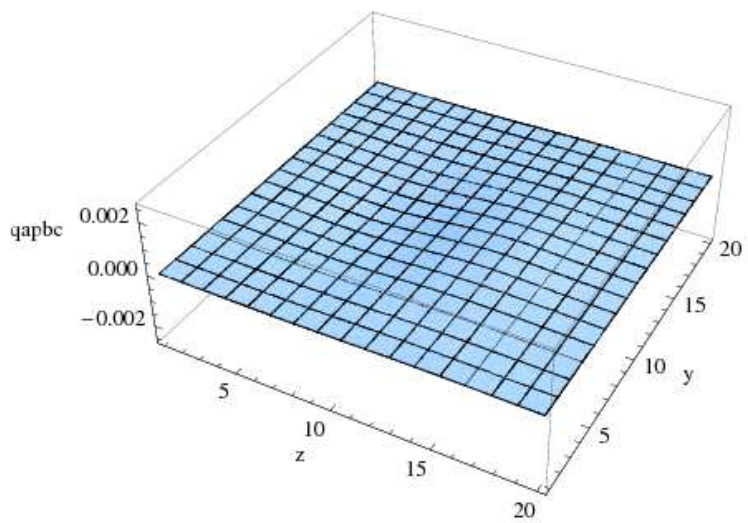

(e)

FIG. 1: (a) The gluonic topological charge density and (b) the local Polyakov loop for an artificially constructed dyon-antidyon pair. The spectrum (c) for overlap fermions with periodic (left) and antiperiodic (right) boundary conditions (restricted to 20 modes). Inside the circle around the origin the plot is 10 times magnified. The fermionic topological charge density from these 20 lowest modes is shown for periodic (d) and antiperiodic (e) boundary conditions. The latter choice is blind for these constituents. 


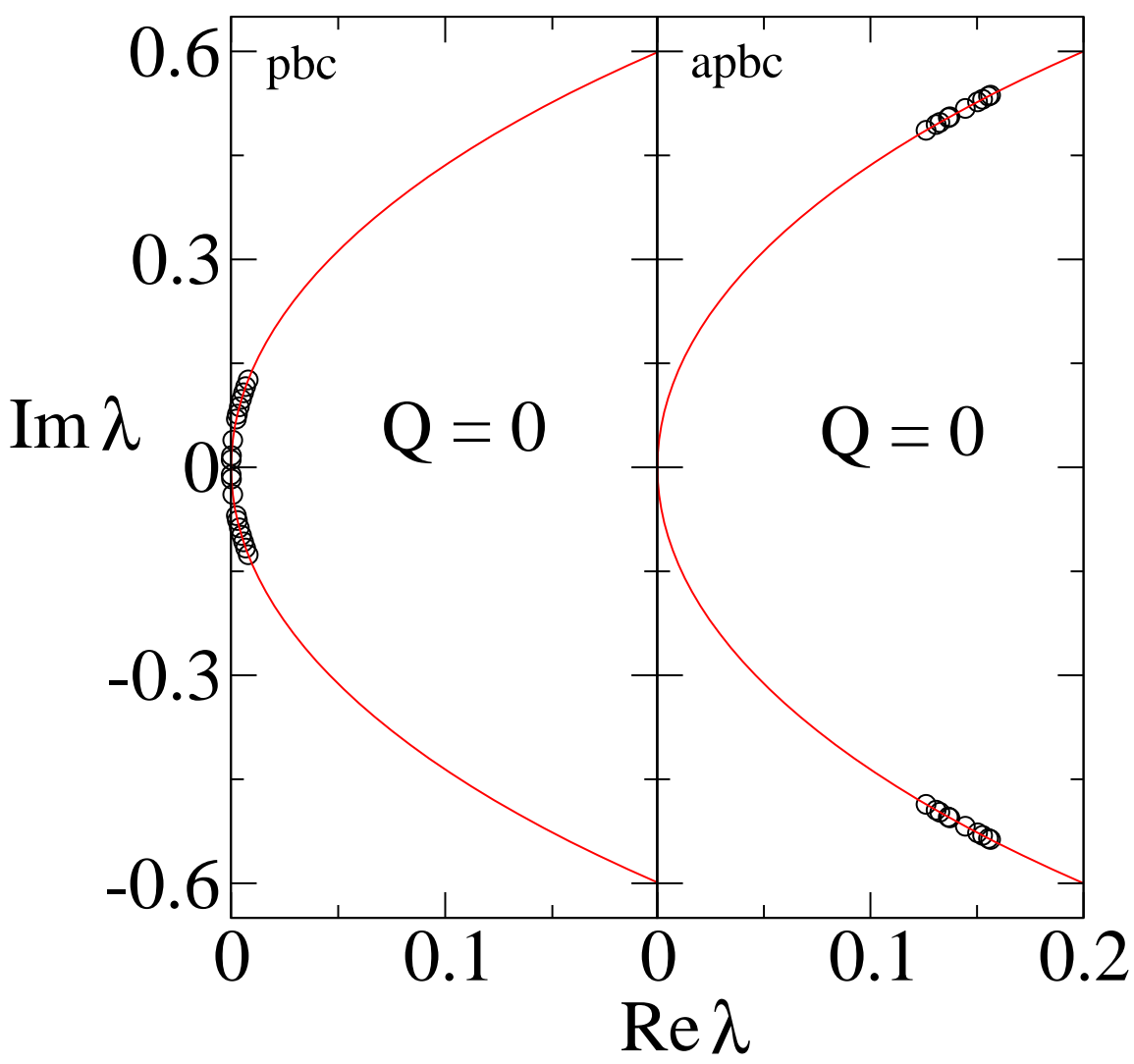

FIG. 2: Periodic (left) and antiperiodic (right) overlap fermion spectra (including 20 modes) for one typical equilibrium MonteCarlo configuration created in the deconfined phase with a positive average Polyakov loop $\langle L\rangle>0$.

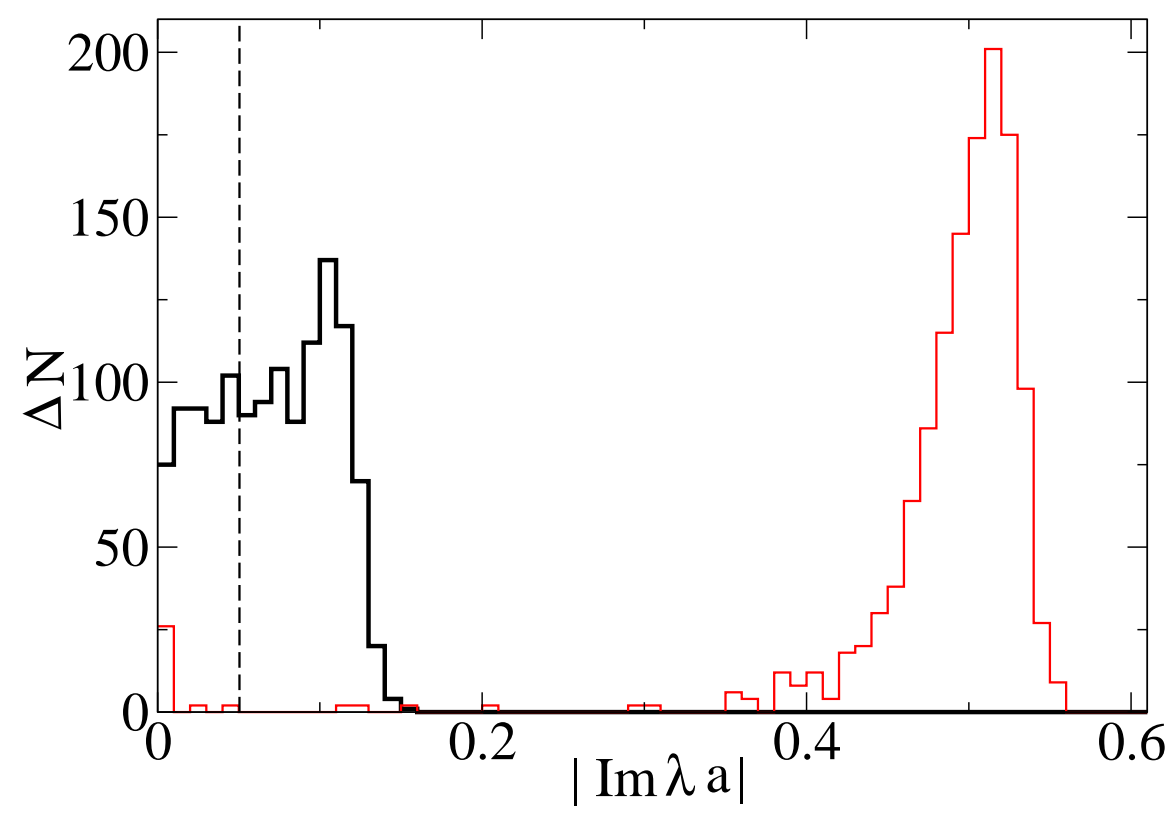

FIG. 3: Overlap fermion spectra for periodic (black thick histogram) and for antiperiodic (red thin histogram) boundary conditions obtained from 67 equilibrium Monte-Carlo configuration (all with positive averaged Polyakov loop) in the deconfined phase. Shown are $(20 \times 67-54)$ periodic nonzero modes and $(20 \times 67-52)$ antiperiodic nonzero modes. The vertical dashed line shows the cut on near-zero modes, see the text for explanation. 


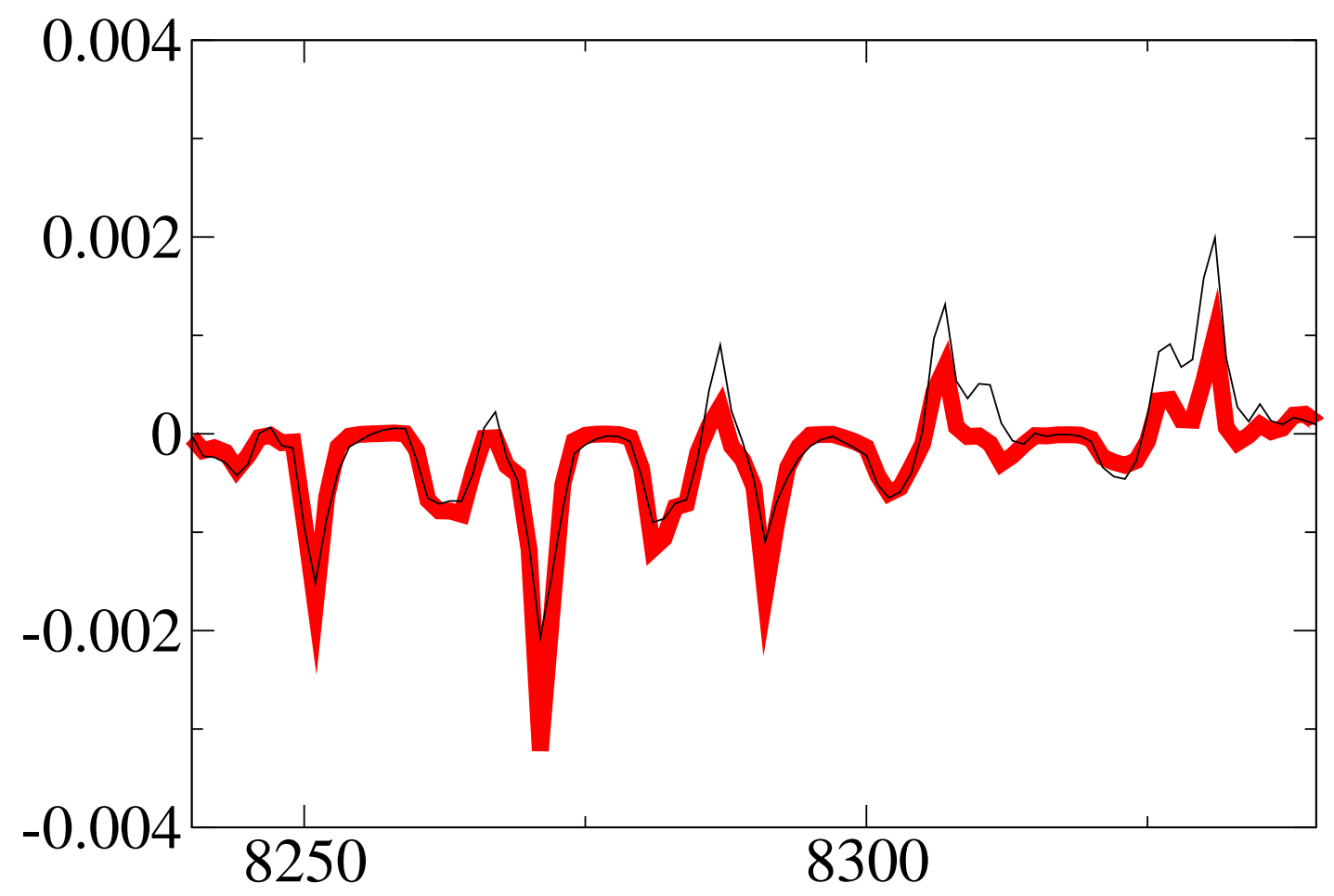

FIG. 4: The topological charge density $q_{\lambda_{\text {cut }}}^{(p)}(x)$ (thin line) and the rescaled topological charge density $q_{\lambda_{\text {cut }}}^{(p)}(x)$ (thick line) for subsequent lattice sites along the $x$-axis in a typical lattice configuration. 


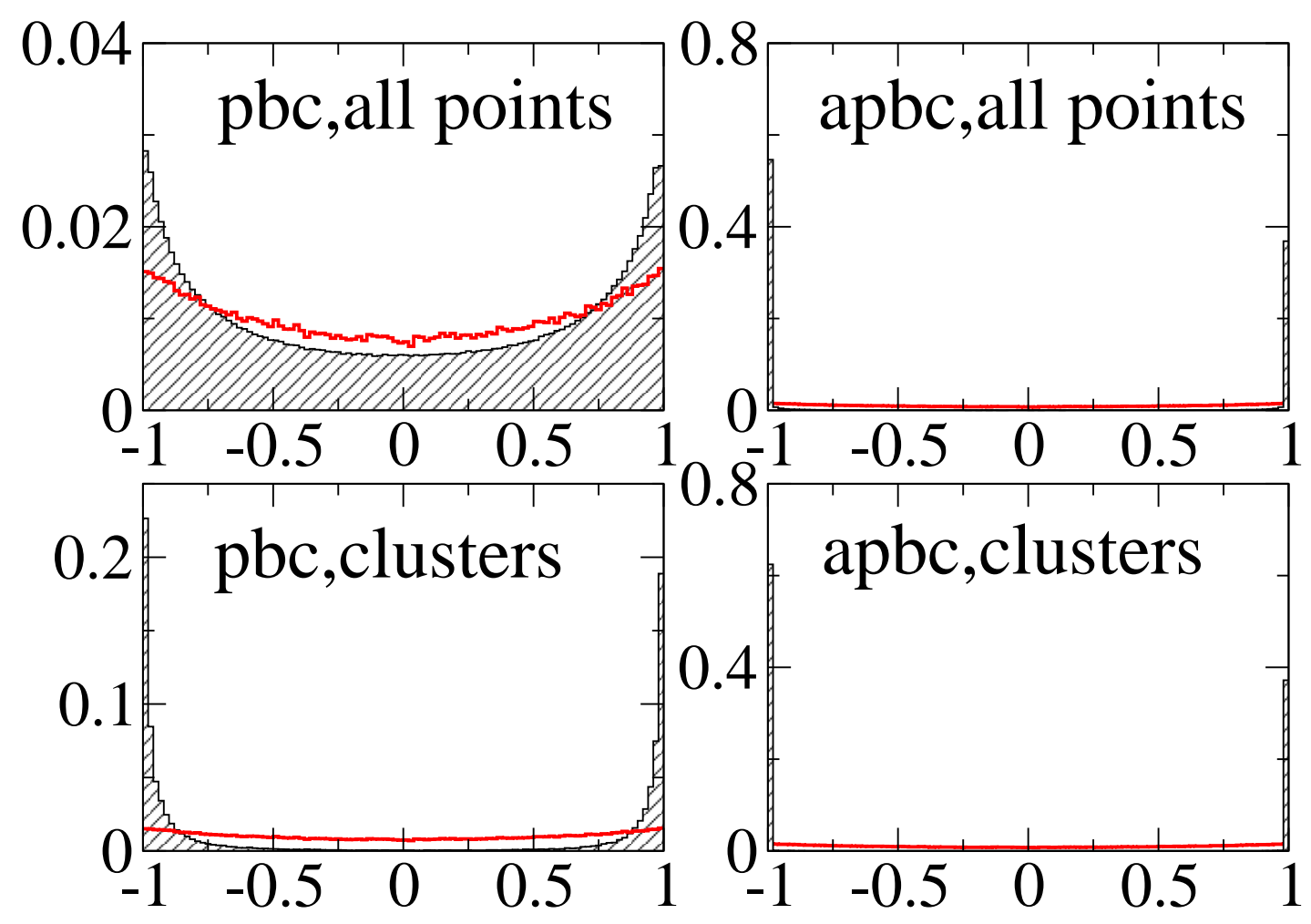

FIG. 5: Histograms with respect to the (anti)selfduality $R$ (8) of all lattice sites (upper row) and within the interior of topological charge clusters (bottom row). Left column: the 20 lowest modes with periodic boundary conditions have been used for the construction of the UV filtered topological density (4) and the UV filtered field strength tensor (5). Right column: only zero and near-zero modes (below the gap) for antiperiodic boundary conditions have been used in constructing the UV filtered quantities. In this case the reconstructed field strength in all sites is either selfdual or antiselfdual, whether they belong to topological clusters (with more than $1 / 5$ of the maximal density) or not. The fat line (red in the colored version) shows the histograms refering to a random assignment of $R$ (see text). 


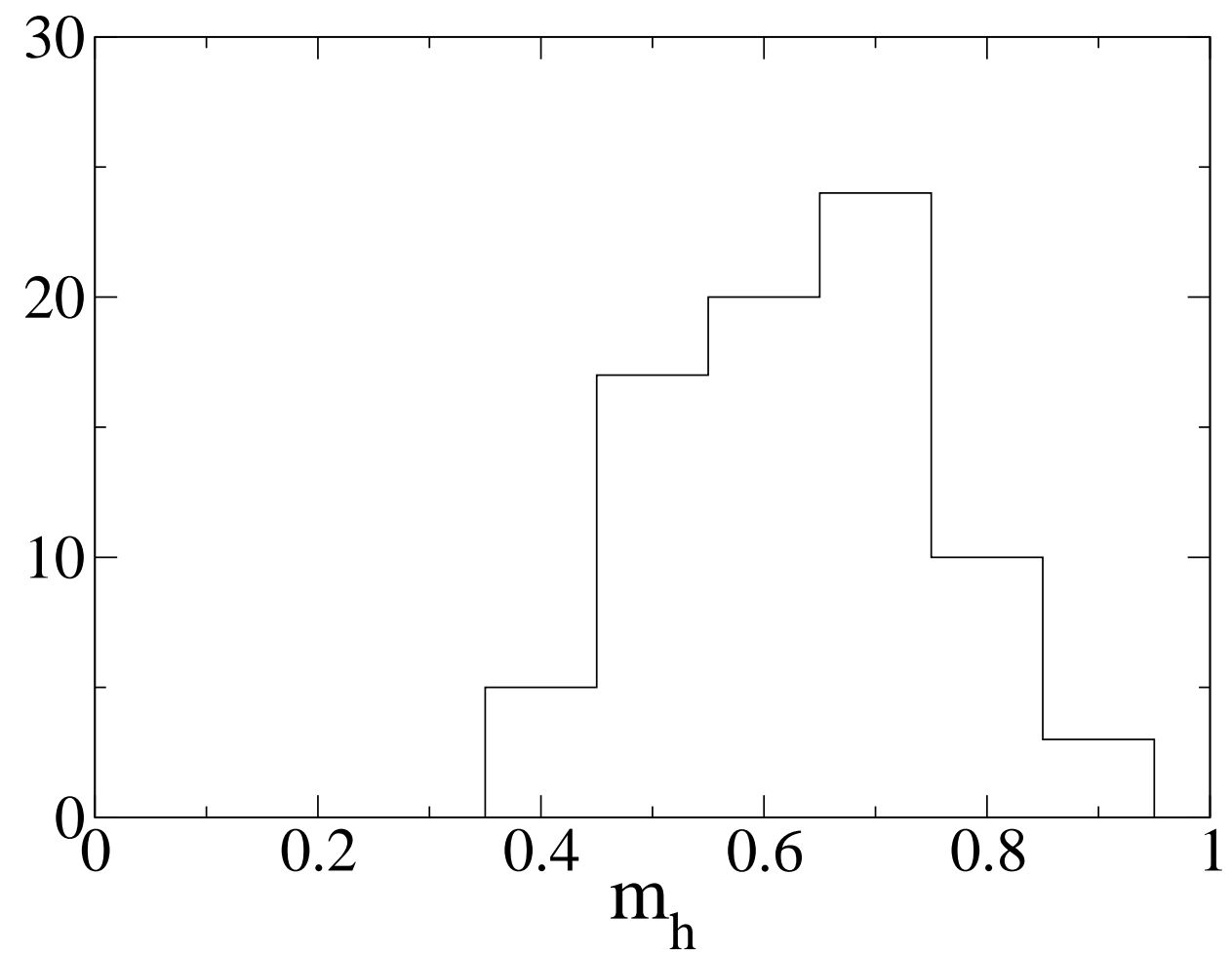

FIG. 6: The distribution of the fractional action (modulus of topological charge) concentrated in heavy dyons, in our simulation represented by the 82 zero and near-zero modes for antiperiodic boundary conditions. Our ensemble has positive average Polyakov loop $\langle L\rangle>0$.

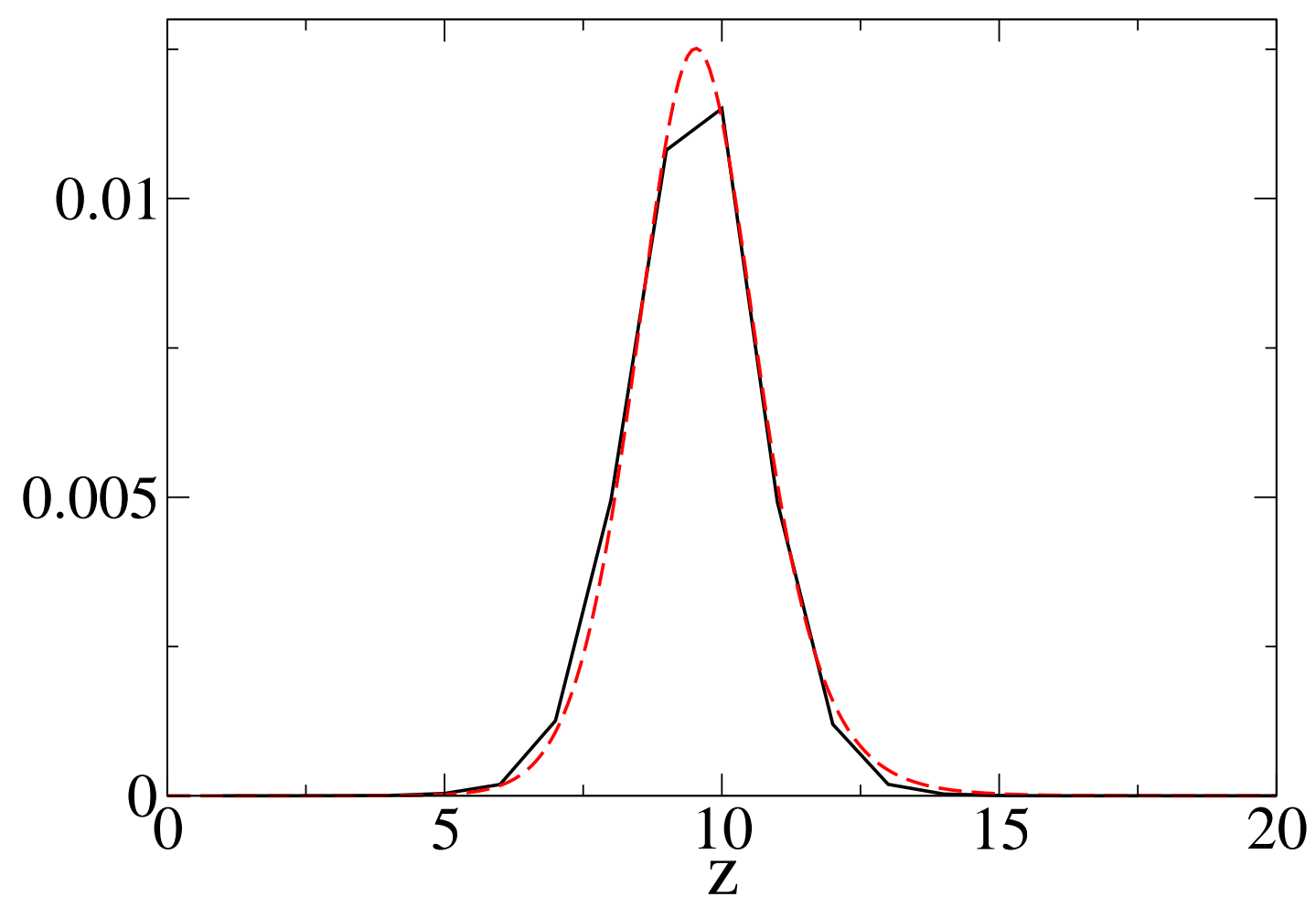

FIG. 7: The scalar density profile (one-dimensional cut) of a zero mode representing one of the 82 heavy dyons (solid line) fitted by the analytical expression for the scalar density of a dyon's fermion zero mode (dashed line). 


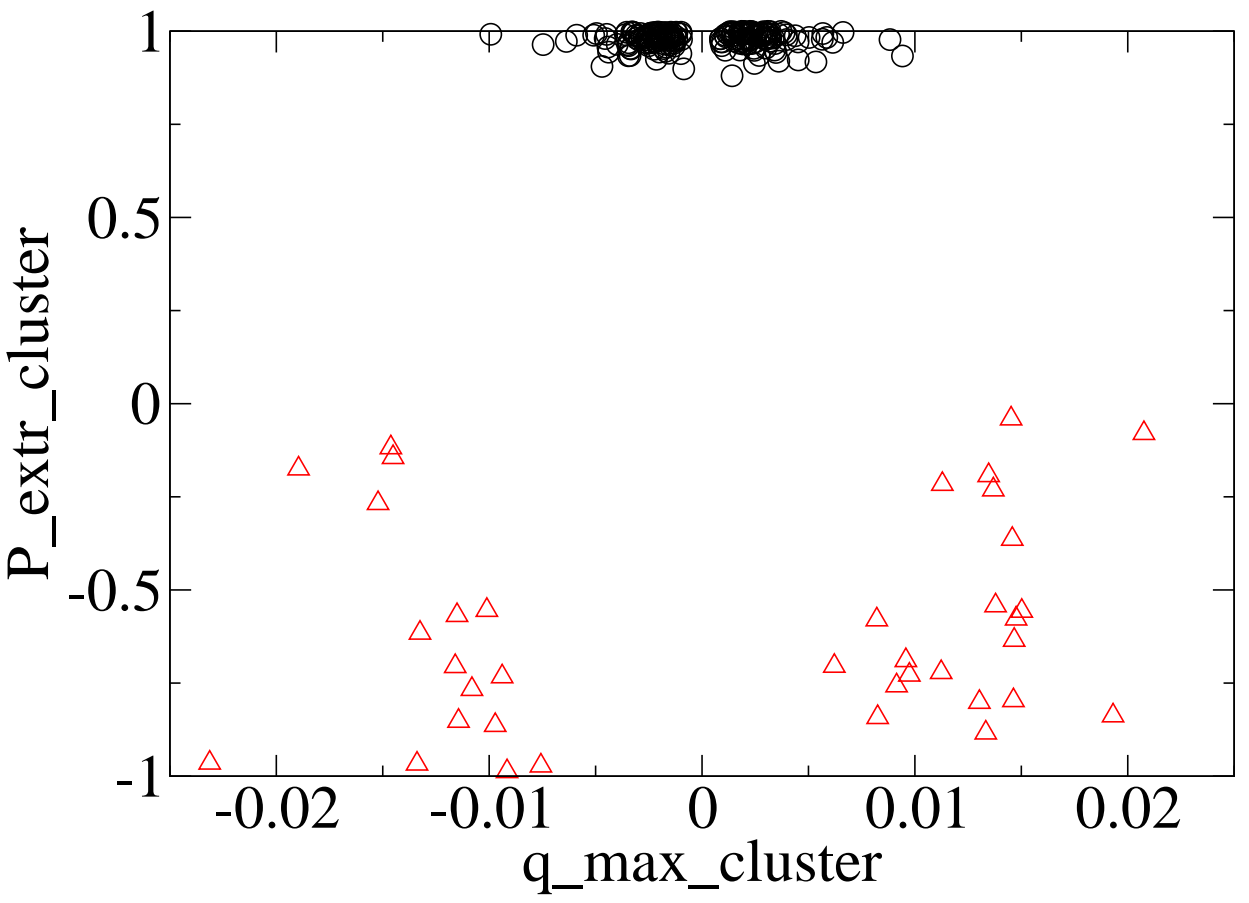

FIG. 8: Clusters containing static monopoles are shown in a scatter plot with respect to the extremal value of the topological charge density and the peak value of the local Polyakov line (inside the clusters, including the sign). Circles correspond to clusters found by periodic fermions (light dyons), triangles correspond to clusters found by antiperiodic fermions (heavy dyons). 